\title{
EVENT INFLUENCED GROUPING PLAN AND VITALITY EFFECTIVE STEERING FOR WIRELESS SENSOR SYSTEM
}

\author{
K.Hari Krishna ${ }^{1}$, Tapas Kumar ${ }^{2}$, Y. Suresh Babu ${ }^{3}$ \\ ${ }^{1}$ Ph.D -Research Scholar-Lingaya's university \\ ${ }^{2}$ Professor \& Dean \& H.O.D, Dept. Of Computer Science \& Engineering, Lingaya's University, Faridabad \\ ${ }^{3}$ Professor, P.G Dept of Computer Science, JKC College, Guntur
}

\begin{abstract}
Remote Sensor systems are gathering of immense number of sensor hubs that sense the ecological and physical conditions, for example, temperature, weight, moistness, sound and so on and send it to the sink. Sensor hubs in the remote sensor system have restricted capacity, preparing, transmitted abilities and additionally restricted vitality sources. Sensor hubs have restricted vitality and asset imperatives so productive usage of vitality is crucial for broadening sensor system life span. Bunching is a compelling procedure in drawing out the lifetime of the WSN.
\end{abstract}

As sensor hubs sense the information, process it and exchange it to the base station, there are odds of repetitive information send by the neighboring hubs. The unavoidable issue is that in vast sensor arranges, the measure of information created is tremendous for the base station to prepare. So routines like information collection are required that consolidate detected information into superb data which prompt vitality protection by diminishing the number of bundles transmitted to basestation.

Keywords-Steering Convention, Bunching in-System Conglomeration, Remote Sensor Systems

\section{INTRODUCTION}

Remote Sensor System (WSN) is at the highest priority on the rundown of the most essential innovations of $\mathrm{t} w \mathrm{e} n \mathrm{t} y$ firstcentury. Remote Sensor System as name recommend is a remote system comprise of little low power gadgets called hubs which making utilization of sensors for detecting their encompassing conditions and correspond with one another in the system through a remote medium.

The quantity of Sensor hubs in remote sensor system might be hundreds or thousands. Sensor hubs are outfitted with sensors; there are distinctive sensors, for example, warm sensors for measuring temperature, mouthpiece sensor for measuring sound, accelerometer, weight sensors and so forth. The elements of these sensor hubs incorporate minimal effort, little size, low power, short range correspondence, multifunctional in term of performing detecting, preparing, directing and so on. Presently days Remote sensor system utilized as a part of numerous nonmilitary personnel applications, for example, in wellbeing, environment, home, space investigation, fiasco help, concoction handling, and other business zones.

The fundamental attributes of WSN incorporate thickly hub organization, restricted battery fueled hubs arrangement, application particular, irregular topology change, selfconfiguration and so forth. A sensor hub in remote sensor system has constrained vitality for performing different procedures, for example, detecting, handling, correspondence and capacity and once in a while it get to be hard to supplant or energize batteries so control must be utilized proficiently [1].
Remote sensor system contain a huge number of sensor hubs sent in intrigued geological territory and a base station that is situated inside or close to the detecting district. Sensor hubs sent in range of enthusiasm for WSN sense the information what's more, exchange it to the base station. Vitality utilization of hubs in remote sensor system for sending the information to the base station is much higher than other assignment such as detecting and handling. The correspondence between base station and sensor hubs can happen either in level or hierarchal style. Hierarchal construction modeling otherwise called bunching in which sensor hubs initially gathered into group and a higher vitality hub from every group is chosen as a bunch head. Sensor hubs send their information to the base station through distinctive group heads.

Every bunch head total the information send by their neighbor hubs. Information accumulation is imperative in WSN in light of the fact that sensor hubs are thickly conveyed in remote sensor system and there is immense probability that diverse hubs send same information to the bunch head. In level Sensor hubs send their information straightforwardly to the base station.

Hierarchal structural engineering expended less vitality than level in light of the fact that in hierarchal information is send over a short separation. Remote sensor system are utilized as a part of numerous applications such as military, Natural, territory observing, building checking, wellbeing observing and so forth. In numerousapplications, little sensor hubs are thickly sent in intrigued topographical range either in adhoc or in pre-arranged way also, left unattended to sense 
parameters like moistness, weight, speed, temperature and so on information send by these sensors are gathered by the base station(observer). Unattended nature of WSNs makes it hard to revive batteries of the hubs. Vitality proficiency is a noteworthy outline objective of WSNs. A few applications require just collected information to diminish correspondence overhead e.g. in natural surroundings observing normal reported moistness worth is adequate for the sink.

Information accumulation lessens the system overhead which brings about vitality investment funds [2]. Different examination thinks about demonstrate that proficient system association, hubs assembled into bunches bolster information collection. Bunching component make a change in Remote sensor system in lessening vitality utilization andoverhead.

\section{BUNCHING PROCESS IN WSNS}

Remote sensor system made out of a huge number of sensor hub sent either in predefined or in adhoc conduct. Every sensor hub in the system is proficient in detecting, handling and transmitting the information to the sink by means of remote channel.

Sensor hubs are furnished with little fueled battery, handset, and handling and correspondence unit. Hub has restricted vitality and it can't be energized effortlessly so exceptional consideration must be made tolow power utilization. Numerous inquires about demonstrate that bunching/ hierarchal system increment system execution than a level system. In hierarchal system, sensor hubs are gathering together to structure groups and a hub from every bunches which fulfilling assessment criteria, for example, high got signal quality and high vitality level is chosen as a group head $(\mathrm{CH})$. $\mathrm{CH}$ not just total the information send by the hubs in the group additionally go about as a controller to make different directing and booking criteria [2].

Progressive grouped sensor systems can be isolated intotwoclassifications:homogeneous and heterogeneous.

Bunching procedure is partitioned into two sections:

1) Bunchdevelopment.

2) Bunch headchoice.

\subsection{Bunch Development Stage}

This is the first stage in bunching. In this stage entire sensor hubs in the system are organize into gatherings and a gathering of hubs in the system is called bunch. Bunch head choice stage: In this stage a hub from every bunch is chosen as a group head $(\mathrm{CH})$ in light of an assessment criteria, for example, high vitality hub is chosen as a bunch head $(\mathrm{CH})$ Bunch based conventions gather the remote sensor hubs into bunches and give numerous favorable circumstances to the WSNs for example, appropriating vitality equally, decreasing overhead, lessening information excess and enhance system life span. Bunching conventions are separated into two classes: 1) pioneer first approach and 2) bunch first approach. In group initially approach sensor hubs first frame groups and afterward bunch head is chosen. In pioneer first approach bunch head is chosen first on the bases of specific measurements and at that point hubs are doling out to every group head. Time driven and Occasion driven directing are two methodologies in hierarchal group based directing conventions.

In time driven conventions sensor hubs send the detected information to the base station intermittently yet in occasion driven steering conventions sensor hubs send the information to the base station just when an occasion is recognized. Remote sensor systems cover a huge land region. Sensor hubs send their information to the base station either through single jump or multi-bounce. For all intents and purposes it is difficult to have an one jump directing model in which every hub specifically send information to the base station. Vitality level of sensor hubs diminishes rapidly as a result of substantial transmission separation.

Hierarchal directing is a decent alternative to manage such issues in which substantial separation is secured by utilizing multi-jump correspondence. Hubs impart over little separation by utilizing hierarchal structural planning, henceforth diminishes vitality utilization. There are numerous focal points of grouping are[3]:

1. It spares a great deal of vitality of the hub that sends the information by lessening the separations went by theinformation.

1. In bunching group head perform information collection handle and diminish the measure of excessinformation.

2. It additionally lessens channeldispute.

3. Grouping lessens parcelimpact.

4. Grouping result in better throughput of the system under highload.

\section{INFORMATION CONGLOMERATION}

Sensor hubs are nearly sent in remote sensor system that implies sensor hubs may create much similar information of the detected parameters. Excess information produce by the two or more hubs increment organize overhead, bundle crash. Every one of these truths engages information conglomeration strategy.

In information accumulation strategy information gathered by the sensor hubs in the group is collected at a point known as group head. As of right now all excess information are uprooted and exchange the information to other bunch heads $(\mathrm{CH})$ in the system that consolidate the information with its own particular information and uproot repetitive information and exchange it to the sink. This can diminish restricted activity in individual gathering furthermore decreases worldwide information. In WSNs sensor hubs are normally battery restricted and asset compelled. Keeping in mind the end goal to spare assets also,vitality information must be accumulated in order to maintain a strategic distance from system activity, bundle impact, blockage and so on. The 
point of information conglomeration is to decrease repetitive information transmission in the system.

A noteworthy vitality sparing component for evacuating excess information is in-system total. Crude detected information gathered by the sensor hubs in the system is forward to the sink for handling. The primary point of insystem information conglomeration is to uproot pointless information transmission by uprooting excess information. The outline of a proficient information conglomeration convention is a testing assignment.

Information accumulation methods are firmly combined with how bundle is steered through the system. Remote sensor building design is expected as a vital part in the execution of information accumulation convention. Information conglomeration and parcel directing is done all the while by numerous conventions[4].

\section{CROSS BREED VITALITY PRODUCTIVE APPROPRIATED CONVENTION}

The fundamental necessity of sensor system is to increment system lifetime. Information collection system helps a ton in enhancing system life. Regard utilizes leftover vitality level as essential parameter for selecting bunch head. In instance of tie where two or modes have same remaining vitality also, are equipped for being a bunch head, system topology highlights e.g. hub ID, hub degree, separations to neighbor areconsidered as an auxiliary parameters.

Sensor hubs are of two sorts: homogeneous hubs and heterogeneous hubs. Homogeneous hubs are those which have same introductory vitality level and heterogeneous hubs are those which have diverse introductory vitality level. In this all hubs in the system are thought to be homogeneous having same vitality level [5]. Regard (Cross breed Vitality Proficient Disseminated) convention utilizes the idea of grouping. Bunching can be to a great degree compelling in one-to many, numerous to-one, one-to-any, or one-to-all (telecast) correspondence. Notice has four essential targets:

1. Distributing so as to drag out system lifetime vitalityutilization.

2. Ending the grouping process inside of a steady number ofemphases.

3. Minimizingcontrol.

4. Creating very much circulated groupheads.

\section{TREE BASED METHODOLOGY}

Numerous remote sensor system conventions are typically based on hierarchal association of hubs in the system. The most effortless approach to total information sending from source to destination is to allocate a few hubs in the course as a total point known as aggregator. In these conventions tree structure is built first in which root is spoken to as sink and sensor hubs are alluded as takes off. Information total began when ahubgets two or more information bundles, this hub then total all the information gathered from the hubs with its own particular information and evacuate excess information.

Aggregator sends one and only information bundle to the hub present beneath the aggregator in the tree. Tree based approach as likewise a few downsides. For instance when information is lost at certain level of tree, information is lost from the entirety subtree.

\section{CONCLUSION}

In this paper, an outline of secure information accumulation idea in remote sensor systems has been introduced. This paper introduces that remote sensor system comprises of gigantic number of sensor hub having detecting, preparing what's moreand correspondence abilities. These hubs are asset limitation. Hierarchal construction modeling is useful for system life span. That is the reason lifetime of the system is restricted so the different methodologies or convention has been proposed for expanding the lifetime of the remote sensorsystem.

\section{REFERENCES}

[1]. Nayyar, Anand, and Agam Gupta. "A Comprehensive Review ofCluster-Based Energy Efficient Routing Protocols in Wireless Sensor Networks."IJRCCT 3.1 (2014): 104-110. [2]. Ma, Ming, and Yuanyuan Yang. "Clustering and load balancing inhybrid sensor networks with mobile cluster heads."Proceedings ofthe 3rd international conference on Quality of service inheterogeneous wired/wireless networks.ACM, 2006.

[3]. Younis, Ossama, Marwan Krunz, and SrinivasanRamasubramanian."Node clustering in wireless sensor networks: recent developments and deployment challenges."Network, IEEE 20.3 (2006): 20-25.

[4]. LanTien Nguyen, Xavier Defago, RazvanBeuran , Yoichi Shinoda,"An Energy Efficient Routing Scheme for Mobile Wireless Sensor Networks"

[5]. Kour, Harneet, and Ajay K. Sharma. "Hybrid energy efficientdistributed protocol for heterogeneous wireless sensornetwork."International Journal of Computer Applications 4.6(2010): 0975-8887.

[6]. KiranMaraiya, Kamal Kant, Nitin Gupta, "Wireless Sensor Network:A Review on Data Aggregation", International Journal of Scientific \& Engineering Research Volume 2, Issue 4, April -2011 ISSN 2229-5518, IJSER 2011.

[7]. HarneetKour, Ajay K. Sharma, "Hybrid Energy EfficientDistributed Protocol for Heterogeneous Wireless Sensor Network", International Journal of Computer Applications (0975-8887)Volume 4-No.6, July 2010.

[8]. OssamaYounis and Sonia Fahmy, "HEED: A Hybrid, Energy-Efficient, and Distributed Clustering Approach for Ad-hoc Sensor Networks".

[9]. M. BHEEMALINGAIAH, M. M.NAIDU,D. Sreenivasa RAO, "Energy Aware Clustered Based Multipath Routing in Mobile Ad Hoc Networks", I. J. Communications, Network and SystemSciences, 2009, 2, 91-168, Published Online May 2009 in SciRes. 
[10]. Leandro Villas, AzzedineBoukerche, Heitor S. Ramos, HoracioA.B. F. de Oliveira, Regina B. de Araujo and Antonio A. F. Loureiro,"DRINA: A Lightweight and Reliable Routing Approach for in-Network Aggregation in Wireless Sensor Networks", 2012 IEEE.

[11]. D.D.Chaudhary, PranavPawar, Dr. L.M. Waghmare, "Comparison and Performance Evaluation of Wireless Sensor Network with different Routing Protocols", 2011 International Conference on Information and Electronics Engineering, IPCSIT vol.6 (2011), IACSIT Press, Singapore.

[12]. Kemal Akkaya and Mohamed Younis, Moustafa Youssef, "EfficientAggregation of Delay-Constrained Data in Wireless Sensor Networks".

[13]. Ramesh Rajagopalan, Pramod K. Varshaney, "Data aggregation techniques in sensor networks: A survey".

[14]. KamanashisBiswas, VallipuramMuthukkumarasamy, ElankayerSithirasenan, "Maximal Clique Based Clustering Scheme for Wireless Sensor Networks", IEEE AmandeepKaur et al, / (IJCSIT) International Journal of Computer Science and Information Technologies, Vol. 5 (4) , 2014.

\section{BIOGRAPHIES}

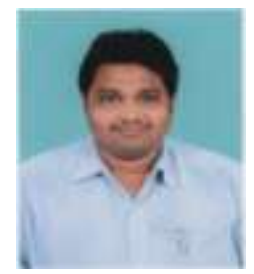

KONDA. HARI KRISHNA received his $\mathrm{M}$.TECH in computer science from Jawaharlal Nehru Technological University, Kakinada \& A.P and pursuing Ph.D in LINGAYA's University, Faridabad. He is working as an Assistant Professor in Bharat Institute of Engineering \& Technology in Dept. of Computer Science \& Engineering. He published 10 Research Papers in Various International Journals of Reputed and His Research Area is Mining of applications in Wireless Sensor Networks. He is a good researcher\& who has worked mostly on WirelessSensor networks,Ad hoc\& Wireless Networks,Network securityand Data mining.

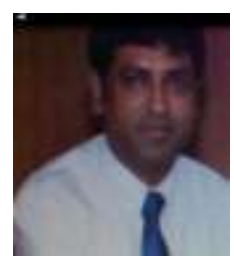

Dr. TAPAS KUMAR, Working as a Professor, Dean \& H.O.D inSchool of Computer Science \& Engineering,Lingaya's University, Faridabad. He holds a Doctorate in Computer Science \& Engineering. He has more than experience of 15 years in Academics \& Administration. He has publishedvarious Research papers in various National \& International Journals of Reputed.

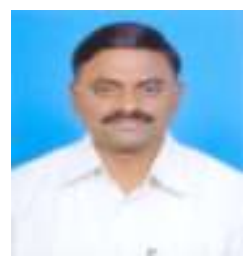

Dr.Y. SURESH BABU, Working as a Professor in Dept of Computer Science, JKC College, Guntur.He holds a Doctorate in Computer Science \&Engg, Image processing as specialization with a combined experience of 23 years in Academics \&Administration. He has published various research papers in various National and International Journals of reputed. 